\title{
Evaluation of Proptosis: A Primer
}

\author{
Syed Shoeb Ahmad, ${ }^{1}$ Ramsha Anwar, ${ }^{2}$ Md Aleemullah Khan, ${ }^{2}$ Nazmi Usmani ${ }^{2}$ and Sadaf Jahan ${ }^{2}$ \\ 1. Ibn Sina Academy, Aligarh, India; 2. Ajmal Khan Tibbiya College, Aligarh, India
}

DOI: https://doi.org/10.17925/OPHT.2021.15.1.26

$P$ roptosis is an abnormal prominence of the eyeball beyond the confines of the bony orbit. It can appear in various systemic, as well as orbital or peri-orbital, disorders. Not only is proptosis potentially vision-threatening, but also it could be a manifestation of lifethreatening conditions, such as metastatic carcinomas. Therefore, management of proptosis is of vital importance. The objective of this review is to present an overview of the systematic evaluation of a case of proptosis and simplify the overall management of this condition. The article comprehensively deals with the causes and classification of proptosis, the problems encountered with the development of the condition, evaluation of a case, and highlights the relevant investigations. The methods for investigating proptosis have been classified into primary, secondary, pathological and laboratory techniques. The article extensively discusses thyroid eye disease, including the current hypothesis of pathophysiology, mechanisms, stages, clinical features, classification and management of this disease. Literature for this review was obtained from textbooks, online databases and search engines, as well as other educative materials available to the authors during the preparation of this review.

\section{Keywords}

Exophthalmos, orbit, Graves' disease, surgical decompression, diplopia

Disclosures: Syed Shoeb Ahmad, Ramsha Anwar, Md Aleemullah Khan, Nazmi Usmani and Sadaf Jahan have no financial or non-financial relationships or activities to declare in relation to this article.

Acknowledgements: The authors would like to acknowledge Prof. Dr. S. Zaheer-ul-Islam, for his invaluable suggestions in the development of this article.

Review process: Double-blind peer review.

Compliance with ethics: This study involves a review of the literature and did not involve any studies with human or animal subjects performed by any of the authors.

Authorship: The named authors meet the International Committee of Medical Journal Editors (ICMJE) criteria for authorship of this manuscript, take responsibility for the integrity of the work as a whole, and have given final approval for the version to be published.

Access: This article is freely accessible at touchOPHTHALMOLOGY.com @ Touch Medical Media 2021.

Received: 9 August 2020

Accepted: 19 January 2021

Published online: 21 May 2021

Citation: touchREVIEWS in Ophthalmology. 2021; 15(1):26-32

Corresponding author: Syed Shoeb Ahmad. IbnSina Academy, Dodhpur, 202001, Aligarh India. E: syedshoebahmad@yahoo.com

Support: No funding was received for the publication of this article.
An abnormal protrusion of the eyeball, beyond the boundaries of the bony orbit, is termed 'proptosis'. A similar appearance, seen in endocrine dysfunction, especially thyroid disorders, is called 'exophthalmos'. These conditions are frequently encountered in clinical practice, and for students, residents and trainees, the situation may prove rather challenging. This primer presents a concise blueprint to aid in the evaluation of a case of proptosis. The article also provides an overview of thyroid eye disease (TED), the most common cause of proptosis in usual clinical settings. ${ }^{1-4}$

The bony orbit is virtually a closed socket for the eyeball. It is the cavity, or socket, of the skull in which the eye and its appendages are situated. It is enclosed from all sides except anteriorly. Incidentally, the term 'orbit' can also imply the contents of the bony structure. Another term related to proptosis is 'exorbitism', a condition in which there is a decrease in the capacity of the bony orbit, with the angle between the lateral orbital walls being more than 90 degrees. This leads to a shallow orbital depth but normal orbital content volume. Such a situation causes proptosis in certain congenital disorders of the orbit., ${ }^{3,5}$

Because of the relatively small amount of space between the orbital walls and the eyeball, an expanding lesion within the orbit, or a shallow bony orbit, as often seen in congenital disorders, causes protrusion of the eyeball forwards. Due to inherent weaknesses in the walls of the orbit, diseases of surrounding structures may extend into the orbital cavity easily. Thus, depending upon the location of the lesions impinging upon the orbit, the eyeball may get displaced from its normal position. The term 'dystopia' indicates the displacement of the eyeball in the coronal plane. A number of conditions may lead to this situation, including extraconal masses, craniofacial scoliosis complex, uncorrected unilateral or assymetric bilateral coronal craniosynostosis, facial clefting syndromes and trauma. ${ }^{6.7}$

Evaluation of a case of proptosis requires fine knowledge of the anatomy of the bony orbit and eyeball, the surrounding structures, salient features of proptosis, causes, examination, investigations, differential diagnosis and management. The objective of this review is to compile the important steps in the evaluation of a case of proptosis. The article also incorporates an overview on thyroid orbitopathy. The literature for this article was obtained from online searches of articles related to proptosis, books and course material during the preparation for this article.

\section{Applied anatomy}

The orbits are bony cavities that enclose structures such as the eyeball, extraocular muscles, nerves, blood vessels and a cushion of fat. The orbits are pear-shaped, tapering posteriorly to the apex. The distance between the back of the globe and optic foramen is $18 \mathrm{~mm}$. Conversely, the length of the orbital segment of the optic nerve is comparatively longer, usually in the range of 
Table 1: Bones forming the walls of the orbit

\begin{tabular}{|c|c|}
\hline Wall & Bones \\
\hline Roof (upper wall) & $\begin{array}{l}\text { Frontal; lesser wing of sphenoid } \\
\text { Frontal bone separates the orbit from the anterior cranial } \\
\text { fossa }\end{array}$ \\
\hline Floor (inferior wall) & $\begin{array}{l}\text { Maxilla; palatine; zygomatic } \\
\text { Maxillary bone separates the orbit from the underlying } \\
\text { maxillary sinus }\end{array}$ \\
\hline Medial wall & $\begin{array}{l}\text { Ethmoid; maxilla; lacrimal; sphenoid } \\
\text { Ethmoid bone separates orbit from ethmoid sinus }\end{array}$ \\
\hline Lateral wall & Zygomatic; greater wing of sphenoid \\
\hline Apex & Located at the opening of the superior orbital fissure \\
\hline Base & Forming the anterior part of the orbit \\
\hline
\end{tabular}

25-30 mm. This extra length of the optic nerve and S-shaped configuration allows proptosis to occur to some extent without compromising optic nerve function. The volume of each orbit is about $30 \mathrm{cc}$, approximately one-fifth of which is occupied by the eyeball. 8.9,10

The bony orbit is enclosed by the medial and lateral walls, the roof and the floor on four sides. The four walls converge at the apex of the orbit. The medial is very thin, and is known as 'lamina papyracea'. Therefore, infection from the ethmoidal sinuses can easily reach the orbit. The lateral wall covers the posterior half of the eyeball, leaving the anterior half exposed on this side. However, this wall is the strongest part of the bony orbit. The roof of the orbit is thin and easily damaged by trauma or iatrogenically during surgical procedures. Similarly, the floor of the bony orbit is thin and easily damaged in blow-out fractures of the orbit. Tumours from the maxillary antrum easily invade into the orbit through this pathway. The bones forming the walls of the orbit are given in Table 1.11

\section{Causes of proptosis}

The causes of proptosis are numerous and varied. Broadly, they can be classified into vascular, inflammatory, traumatic, autoimmune, metabolic, iatrogenic, neoplastic, congenital and endocrine (remembered by the mnemonic: VITAMIN CE)..$^{22}$ Vascular causes include infantile haemangiomas, cavernous vascular malformations and direct or indirect carotid-cavernous fistulas. Orbital cellulitis is a type of inflammatory aetiology of proptosis. In at least two studies it was the most common cause of proptosis. ${ }^{2,13}$ Trauma leads to retrobulbar haemorrhage and haematoma formation, which can cause rapid onset of proptosis and affect visual acuity from pressure on the optic nerve. Autoimmune disorders causing acute proptosis can range from idiopathic orbital inflammatory syndrome to immunoglobulin $G$ antibody-mediated inflammatory disease. Aggressive intravenous rehydration therapy can cause orbital compartment syndrome, regarded as a metabolic cause of proptosis. Less common causes include iatrogenically induced proptosis through orbital implants and fillers. Benign, malignant and metastatic neoplasms are also frequently reported in some studies. $13,1,1,15$

Congenital causes of proptosis include dermoid cysts and encephaloceles; the latter often show expansion on Valsalva manoeuver. TED is the most common cause of endocrine and autoimmune proptosis in adults and children. ${ }^{16,17}$ It leads to slowly progressive development of eye signs, including proptosis. The common causes of proptosis in adults are given in Table 2. 12,13,15,16,18 The differential diagnosis for proptosis in the paediatric age group is given in Table 3.19,20
Table 2: Common causes of proptosis in adults

\begin{tabular}{|l|l|}
\hline Category & Specific conditions \\
\hline Endocrine & Thyroid eye disease \\
\hline Trauma & Facial fracture, soft tissue swelling, retrobulbar haemorrhage \\
\hline Vascular & $\begin{array}{l}\text { Carotid-cavernous fistula, cavernous sinus thrombosis, } \\
\text { cavernous haemangioma }\end{array}$ \\
\hline Inflammatory & $\begin{array}{l}\text { Inflammatory orbital pseudotumor, dacryoadenitis, orbital } \\
\text { myositis, Tolosa-Hunt syndrome, Wegener's granulomatosis, } \\
\text { sinus mucocele, sarcoidosis, Churg-Strauss syndrome }\end{array}$ \\
\hline Infective & $\begin{array}{l}\text { Orbital cellulitis, mucormycosis } \\
\text { Tumours }\end{array}$ \\
\hline $\begin{array}{l}\text { tumour, meningioma, neurofibroma, optic nerve glioma, } \\
\text { metastasis, myeloid sarcoma, ossifying fibroma, orbital } \\
\text { osteoma, haemangioblastoma, neuroblastoma, neurofibroma, } \\
\text { acute leukaemia }\end{array}$ \\
\hline Other & $\begin{array}{l}\text { Paget's disease, fibrous dysplasia, Langerhans cell } \\
\text { histiocytosis, Erdheim-Chester disease }\end{array}$ \\
\hline
\end{tabular}

Table 3: Causes of proptosis in children

\begin{tabular}{|l|l|}
\hline 1. & Thyroid eye disease \\
\hline 2. & Rhabdomyosarcoma \\
\hline 3. & Orbital cellulitis \\
\hline 4. & Optic nerve glioma \\
\hline 5. & Orbital inflammatory disease \\
\hline 6. & Retrobulbar haemorrhage \\
\hline 7. & Chloroma \\
\hline 8. & Neuroblastoma \\
\hline 9. & Histiocytosis \\
\hline
\end{tabular}

\section{Classification based on onset}

Depending upon the onset at which the disease appears, proptosis can be regarded as acute, subacute or chronic. Acute proptosis is defined as one that develops over a few minutes or hours; subacute develops over a few days or weeks; and chronic develops over a few months. Acute and subacute proptosis can occur in conditions like non-specific orbital inflammation, orbital cellulitis, trauma, retrobulbar haemorrhage, thrombophlebitis, and tumours such as rhabdomyosarcoma, neuroblastoma, granulocytic sarcoma and metastasis. Chronic proptosis occurs in diseases, such as fibrous dysplasia, and tumours, such as dermoid cysts, lymphomas, benign mixed tumours, neurogenic and vascular tumours, and osteomas. ${ }^{5,21}$

\section{Direction of proptosis}

Proptosis can be axial, when it occurs in the anteroposterior direction or can be in the direction opposite to the causative lesion. Thus, a lesion in the upper-lateral aspect of the orbit, such as a lacrimal gland tumour, will push the eyeball downwards and medially. Looking at the displacement of the eyeball the probable site and cause of the offending lesion can be surmized. Further examination and investigations would help in arriving at the correct diagnosis. Table 4 provides a list of causes and the direction in which they cause proptosis.

\section{Common problems due to proptosis}

The displacement of the eyeball from its normal position causes a number of sequelae, which include the following:22 
Table 4: Direction and displacement of the eyeball due to different causes

\begin{tabular}{|l|l|}
\hline Direction & Aetiology \\
\hline Axial & $\begin{array}{l}\text { Thyroid eye disease; } \\
\text { Lesions in the muscle cone, e.g. optic nerve glioma, } \\
\text { meningioma, haemangioma }\end{array}$ \\
\hline Down and outward & $\begin{array}{l}\text { Lesion in the upper and inner quadrant of orbit, e.g. } \\
\text { dermoid, dermolipoma or invading lesions from } \\
\text { ethmoid or frontal sinus }\end{array}$ \\
\hline Down and inward & $\begin{array}{l}\text { Lacrimal gland tumour and dermoid in outer orbital } \\
\text { margin }\end{array}$ \\
\hline Outward & $\begin{array}{l}\text { Lesions of anterior ethmoidal sinus and } \\
\text { nasopharyngeal carcinoma }\end{array}$ \\
\hline Upward displacement & Carcinoma of maxillary antrum \\
\hline
\end{tabular}

- Exposure keratopathy: Proptosis is associated with a poor blink mechanism and the inability of the eyelids to completely cover the cornea (lagophthalmos). These mechanisms lead to dryness of the eye and inability to wet the cornea properly, leading to exposure keratopathy. This may lead to an increased propensity to develop corneal abrasions and ulcers. These ulcers have a high risk of perforation and the development of endophthalmitis.

- Diplopia: The displacement of the eyeball and enlargement of the extraocular muscles during the development of proptosis leads to restriction of ocular movements. These phenomena lead to diplopia early during the course of the disease. In later stages, there is fibrosis of the muscles, which causes various degrees of dysfunction. The mechanical changes in the muscles affect their function leading to diplopia.

- Optic nerve compression: The increase in the bulk of tissues behind the eyeball causes pressure on the optic nerve. This leads to a decrease in optic nerve function, characterized by signs such as reduced visual acuity, relative afferent pupillary defect (RAPD), redcolour desaturation and visual field defects.

\section{Evaluation of a patient with proptosis Patient history}

The duration of the disease, concerning the onset and clinical course, is a useful starting point in history taking. As mentioned previously, the onset of proptosis can be acute, subacute or chronic. Acute proptosis is usually seen in inflammatory and infective conditions, such as orbital cellulitis. such conditions normally have a short course, as the body's immune system or treatment usually resolves the cause of proptosis. Conversely, chronic diseases, such as TED; tumours; and chronic infections, such as tuberculosis, may have a more prolonged course. ${ }^{23}$

History of the patient's vision should also be assessed. A history of slowly worsening visual acuity can be attributed to either exposure keratopathy or a slowly developing compressive lesion involving the optic nerve. However, conditions such as orbital cellulitis and retrobulbar haemorrhage can cause an acute decrease in vision by affecting optic nerve or vascular integrity. Another vision-related clue is a history of diplopia, which can give a significant indication of the probable cause of proptosis. Slowly developing diplopia indicates a likely benign condition, such as TED, while a quick onset of diplopia can be seen in orbital cellulitis or haemorrhagic conditions.

The patient should be assessed regarding pain; a painful proptosis occurs in inflammatory and infectious conditions. It also occurs in orbital haemorrhage, malignant tumours of the lacrimal gland or invasive nasopharyngeal carcinoma, and metastatic lesions. ${ }^{5,16}$

Epistaxis/nasal obstruction should also be investigated. In cases of rhinological aetiology of proptosis, there can be symptoms like recurrent epistaxis or nasal obstruction. Furthermore, a history of trauma should be elicited. Trauma is a common cause of proptosis in young adults. ${ }^{15,16}$ It can be associated with haemorrhage, orbital emphysema or intra-orbital foreign bodies.

History of systemic diseases, including family history, should be taken. Several systemic diseases can cause proptosis. These include thyroid disorder, tuberculosis and blood-borne metastasis [Tables 2 and 3]. Finally, any changes in proptosis should be noted. A history of change in the severity of proptosis with posture is seen in conditions such as orbital varix and carotid-cavernous fistulas. ${ }^{16,24}$ The change could also be associated with fractures of the roof of the orbit. However, in that situation, the proptosis may be too subtle to be noted by the patient or attendants.

\section{Examination}

An assessment of visual acuity should be conducted at the first visit and on follow-up visits. If the patient is admitted to the ward, frequent monitoring of visual acuity and colour vision may be required. The mechanisms of visual loss in such scenarios include central retinal artery occlusion, direct compressive optic neuropathy, compression of small nutrient vessels of the optic nerve leading to ischemic optic neuropathy. Stretching of the nerve and posterior globe tenting aggravate the situation. ${ }^{25}$ Vision may also be affected by exposure keratopathy as explained above.

A physical inspection should also be conducted; this should not be limited to the eye. It should include the general appearance of the patient, a cachexic build possibly implying a chronic disease; the appearance of the skull, an abnormality suggesting craniosynostoses; and any swelling over the neck, which could relate to the thyroid gland. Inspection should also be done to determine laterality of the proptosis, whether it is unilateral or bilateral, and direction of the proptosis. Additionally, the clinician should look for signs of acute inflammation, such as peri-orbital and conjunctival congestion and chemosis. A physical examination can also reveal a squint, which may occur in both acute and chronic stages of TED. The appearance of capillary haemangiomas of the lids should be noted, as they produce skin lesions, known as strawberry nevi, which increase in size up to a year after birth. The presence of plexiform neurofibromas of the lids and orbits should also be established; they have a 'bag of worms' appearance and texture. They may also produce an S-shaped curvature of the upper lids. In asymmetrical proptosis, inspection can be done by looking from above and behind the patient. Examination can also be performed by extending the neck of the patient and observing from below. ${ }^{6}$

Many peri-orbital changes can provide clues to the possible diagnosis. Conditions such as mycosis fungoides, lymphangiomas and orbital varices may produce non-specific changes including lid oedema, erythema, ecchymosis and eczema. While appearing innocuous, they often point to deeper pathologies. Similarly, intra-orbital haemangiomas are often associated with superficial lesions such as strawberry nevi. Lymphomas are also associated with salmon patches in the conjunctival fornix, while, corkscrew conjunctival vessels are seen in carotid-cavernous fistulas. ${ }^{16}$

The orbital margin should be palpated for irregularities, swellings and foreign bodies. The little finger is insinuated between the orbital rim and the eyeball to feel for any mass and for its consistency. Any area of 
tenderness should be recorded. A reducibility test can also be performed; this is done by placing the flat of the palm on the closed lids and applying light pressure for a short while and then releasing it. If there is no change in the amount of proptosis, then reducibility is excluded. Reducibility is seen in diffuse inflammation, such as early TED and vascular lesions, including haemangioma, lymphangioma and orbital varices. An increased resistance to reducibility is a non-specific sign. ${ }^{5}$

On inspection, pulsation can be observed, or a bruit felt on palpation of the proptosis. Pulsation, or thrill, occurs when a vascular pulse is transmitted to the orbit. It happens when an abnormal vascular flow creates an area of turbulence. The palm is put on the closed eyelids to feel for the thrill. Minor degrees of pulsation can be difficult to assess. In such cases, a Goldmann Applanation Tonometer can be applied to the cornea and the movement of the mires with each arterial pulsation observed. This would especially be useful in cases of orbital roof fractures.

Auscultation is performed by applying the bell of the stethoscope to listen for sounds over the orbital and mastoid regions. A bruit can be detected over the orbital bones or eyeball in vascular tumours, such as haemangioma, or in carotid-cavernous fistulas. Gentle pressure over the ipsilateral carotid artery in the neck would reduce the bruit and thrill in case of carotid-cavernous fistulas. The patient may complain of hearing an audible bruit or have tinnitus. ${ }^{26}$ Several classical signs are seen in carotid-cavernous fistulas, such as pulsatile proptosis, chemosis, arterialized conjunctival and episcleral vessels, ptosis, ophthalmoplegia, increase in intraocular pressure due to rise in episcleral venous pressure, orbital congestion, and occasionally, angle-closure mechanisms. Retinal changes, such as haemorrhages, disc swelling and venous congestion, may also occur. $6,27,28,29$

Another test is performed by asking the patient to bend forwards and cough. This increases the intraorbital pressure. In case of orbital varix, this action leads to an increase in the degree of proptosis, which can also be demonstrated by performing the Valsalva manoeuvre. ${ }^{16,24}$

Extraocular movements should also be assessed in all cardinal directions of gaze. In lesions situated in the muscle cone and early/mild TED, the movements are unaffected for a considerable period. However, in traumatic, inflammatory or malignant conditions, a rapid restriction of extraocular movements occurs. Haemorrhage in a benign tumour may also cause a sudden onset of ophthalmoplegia. ${ }^{30}$

Finally, ophthalmoscopy should be performed. Fundus findings in cases of proptosis are varied, depend upon the cause and include disc swelling (in cases of raised intracranial pressure), optic atrophy (in cases of primary/secondary optic atrophy), choroidal folds (due to retrobulbar pressure), retinal haemorrhages (in central retinal vein occlusion, traumatic retrobulbar haemorrhage and carotid-cavernous fistulas) and optociliary collaterals (in optic nerve sheath meningioma and optic nerve glioma). ${ }^{6}$

\section{Investigations}

\section{Primary investigations}

The severity of proptosis can be measured by various methods. The Hertel exophthalmometer uses a system of prisms to project a lateral view of the eye forward. A millimetre-scale is superimposed on this view and is read by the opposite eye of the examiner. The patient is made to sit at the eye level of the examiner. The two ends of the instrument are placed at the lateral orbital margins of each eye. Subsequently, the observer uses his opposite eye to look at the distance of the apex of the cornea on the scale by excluding parallax. A millimetre-scale is provided on the top of the instrument to measure the distance between the lateral orbital margins of the two eyes; this reading can be noted for future reference. Errors in measurement are caused by thickness of subcutaneous tissue over the bone, facial asymmetry, parallax and examiner skills.6.31 Apart from Hertel's exophthalmometer, a simple plastic scale at the lateral orbital margin, or a Luedde exophthalmometer, can be used. The latter is a thick plastic ruler with a notch to fit in the lateral orbital margin and markings in millimetres on both sides of the scale to avoid parallax. In exophthalmometry, normal readings are taken as less than $21 \mathrm{~mm}$ between the lateral orbital rim and apex of the cornea, or a difference of less than $2 \mathrm{~mm}$ between the two eyes. ${ }^{1,6}$

A computed tomography (CT) scan is useful in determining the shape, position, extent (including whether the lesion is within the orbit or has extension intra-cranially) and nature of lesions in the orbit. Orbital scans are usually obtained in $3 \mathrm{~mm}$ slices, though 'finer cuts' of $1.5 \mathrm{~mm}$ can be ordered for better vizualisation of orbital pathologies. The CT scan appears to be the technique of choice to visualize bony lesions and erosions in most situations. CT also visualizes calcification efficiently in cases of retinoblastoma. To better locate the lesions, 3D images can be constructed with fine slices of the images. ${ }^{5}$ Axial scans, which must include the lenses on both sides, are useful in identifying proptosis. The distance between the apex of the cornea and a line joining the zygomatic processes (interzygomatic line) is measured. This distance should not exceed $22 \mathrm{~mm}$, or the difference between the two sides should not be more than $2 \mathrm{~mm} .{ }^{32}$ Measurements between the interzygomatic line and the posterior sclera can also be used; proptosis is suggested when less than one-third of the eyeball lies behind this line. ${ }^{33}$ In TED, imaging studies are not mandatory for diagnosis, but they are utilized in cases where a diagnosis is difficult, such as uniocular cases. It is also required where visual obscuration is developing, to assess the optic nerve. In TED, the muscle bellies are characteristically fusiform with sparing of the tendons. ${ }^{6,16,33-35}$

The wide range of orbital pathologies causing proptosis may prove to be diagnostic and management challenges. Imaging techniques, such as magnetic resonance imaging (MRI) provide high-resolution soft tissue details leading to better lesion characterization. This may prove crucial where history and clinical evaluation are equivocal. ${ }^{36}$ These techniques often use a common strategy of localizing the pathologies to one of the defined orbital compartments, namely, muscle cone, extra-conal compartment, lacrimal gland and globe. ${ }^{37}$ MRI is invaluable in determining the extent of disease, describing the exact location of the lesion and detecting involvement of the orbital compartments and intracranial extension. It is also useful in identifying the size of the tumours, extraocular extension and ciliary body infiltration. In early TED, CT and MRI can reveal swelling of extraocular muscles and an increase in the volume of orbital fat content. In active disease, the muscles appear hyperintense in T2-weighted images. ${ }^{38} \mathrm{MRI}$ can also reveal a stretched optic nerve, which is associated with an increased risk for visual loss. ${ }^{34,39}$

Characteristics of orbital lesions can be studied by the formation of $2 \mathrm{D}$ images on ultrasonography. ${ }^{40}$ The technique was found useful in assessment of disease activity in TED; however, it was less sensitive compared to MRI. ${ }^{41}$ Other disadvantages of ultrasonography are the inability to image posterior structures, high intra- and inter-observer variability, and the difficulty to image through bone or air. ${ }^{5}$ InTED, individual muscles can be studied in a short time by this low-cost method, which also avoids dangers from radiation. 


\section{Secondary investigations}

venography allows the visualization of blood flow in the veins. This technique was more commonly used in the past to study vascular lesions. Contrast is injected into the frontal, angular or superior ophthalmic vein, and digital subtraction technologies have been developed to better visualize vascular lesions. On the other hand, arteriography (also called angiography) is useful to study arterial lesions such as aneurysms and arteriovenous malformations. However, since dye is injected directly into an artery there is an inherent risk of neurological and vascular complications. With the development of better technologies, such as CT and MR angiography, it has become possible to digitally study vascular lesions without resorting to the injection of dye into the arteries. However, these techniques are still not superior to direct angiography. ${ }^{5.42}$

Finally, colour Doppler imaging produces ultrasonographic images along with information regarding the direction and velocity of blood flow. This method can be of some use in the evaluation of carotid-cavernous fistulas, orbital varix, orbital tumours, orbital cellulitis and orbital inflammatory conditions. In TED, reduction in superior ophthalmic vein flow has been reported. ${ }^{43}$

\section{Pathological studies}

Biopsy specimens from orbital lesions or masses, causing proptosis, can be sent for frozen section studies. The edge of tumours can be analysed intra-operatively by micrographic techniques, making excision of the lesion as complete as possible. Cell surface marker studies on these specimens can be done to differentiate lymphoid tissues, presence of oestrogen receptors, and in poorly differentiated tumours. In certain cases, fine needle aspiration cytology can be performed. However, in such cases, the risk of haematogenous spread of the tumour should be kept in mind.5.44

\section{Laboratory investigations}

In cases of suspected TED, thyroid function tests such as the T3, T4 and thyroid-stimulating hormone (TSH) levels have to be performed. Combined elevation of T4 and decreased TSH levels are sufficient to diagnose hyperthyroidism. In cases of a high index of suspicion for TED and if the above tests come back negative, then additional tests, such as the thyroid-stimulating immunoglobulins or TSH-receptor antibody test, thyroid-binding inhibitory immunoglobulins or anti-microsomal antibody (also called thyroid peroxidase antibody), need to be performed. Wegener's granulomatosis has to be excluded by anti-neutrophil cytoplasmic antibody serum assay. In suspected sarcoidosis serum angiotensin-converting enzyme and lysozyme assays are required..$^{34}$

\section{Pseudo-proptosis}

A misleading impression of proptosis can occur either due to a false appearance of proptosis, or a true asymmetry between the two eyes, but excludes displacement of the globes by any extraocular lesions. conditions leading to pseudo-proptosis include facial asymmetry, lid retraction (as in facial nerve palsy), asymmetric orbital size (seen in congenital disorders such as Crouzon's syndrome), enlargement of the globe (myopia or buphthalmos), ipsilateral lid retraction, iatrogenic pseudo-proptosis (following misuse of phenylephrine eyedrops) or contralateral enophthalmos, ptosis or a small-sized eye..$^{6,45}$

\section{Thyroid eye disease}

The commonest cause of proptosis in most age groups is TED, also called thyroid-associated orbitopathy or Grave's ophthalmopathy. The condition is frequently associated with hyperthyroidism, where excessive thyroid hormones are produced. This situation is regarded as a non-infectious, inflammatory autoimmune disorder, known as Grave's disease. However,
TED may also develop in euthyroid (ophthalmic Grave's disease) or hypothyroid individuals, or in hyperthyroid individuals at a later period after being successfully treated. Grave's disease reportedly occurs in $0.5-2.0 \%$ of the Caucasian population. ${ }^{46}$

In Grave's disease auto-antibodies develop against thyroid tissue leading to an antigen-antibody reaction. This reaction then causes a cascade of events leading to inflammation, and ultimately, two changes take place:

- There is increased binding of antibodies to TSH receptors present on the epithelial surface of thyroid follicular cells, which leads to increased production of thyroid hormones, known as thyrotoxicosis. ${ }^{34.46}$

- There is physical enlargement of the thyroid gland, known as goitre.

Several risk factors have been identified with the development of TED. These include age above 50, smoking, microvascular disease, prior radioactive iodine treatment, and possibly, a family history of Grave's disease. ${ }^{47-49}$ Studies have reported that Grave's disease occurs in $36 \%$ of first- or second-degree relatives of individuals diagnosed with Grave's disease or autoimmunological thyroid disorders..$^{50}$ Others have reported concordance rates of approximately $20-40 \%$ in monozygotic twins and $>10 \%$ in siblings. ${ }^{46,51}$ TED is six times more common in females, as compared to men, and usually develops in the third to fourth decade of life. ${ }^{52}$ However, the severity of the disease is usually worse in males. ${ }^{34}$ The condition may occur prior to, during or subsequent to the hyperthyroid phase. The severity of TED has no relation to the level of thyrotoxicosis.

Thyrotoxicosis leads to inflammation of the extraocular muscles and increased secretion of glycosaminoglycans. The formation of these polysaccharides leads to a large osmotic gradient in the orbital tissues, with increased fluid accumulation between the fibres of the extraocular muscles. As a result, the muscles swell up by almost eight times, leading to restriction of their activity. Another change brought about in thyrotoxicosis is the increased inflammatory infiltrates, such as lymphocytes; plasma cells; macrophages; and mast cells in the interstitial tissues, orbital fat and lacrimal glands. There are three types of cells implicated in the development and progression of TED. These include B cells, T cells and orbital fibroblasts. B cells occur in early disease and produce antibodies against self-antigens. Orbital fibroblasts recruit and activate T cells, which cause tissue remodelling characteristically seen in TED. Some orbital fibroblasts differentiate into mature adipocytes causing increased volume and expansion of the orbital contents. The extraocular muscle swelling and waterlogging cause an increase in the intra-orbital pressure, which compresses the optic nerve. These pathological changes manifest clinically as exophthalmos, myopathy, soft tissue changes and others.33,34,48

\section{Stages of thyroid eye disease}

TED is divided into two stages: congestive and fibrotic. The congestive or inflammatory stage occurs early in the course of TED. In this stage, the eyes are red and painful. Usually, this condition recedes within 3 years. After a few acute attacks, the eye goes into the second stage known as the fibrotic or quiescent stage. In this condition the eye is white and a painless motility defect is present. . $4,49^{-19}$

A number of classifications have been developed to assess the stage of TED. These include NOSPECS, developed by the American Thyroid Association; the European Group on Graves' Orbitopathy (EUGOGO); VISA (vision, inflammation, strabismus, appearance); and the Clinical Activity Score (CAS) ${ }^{6.52}$ The CAS is given in Table 5. In this classification, each criterion is given 1 point for a maximum of 10 points. A clinically significant active disease is regarded as one with a score of more than $4 .^{53}$ 
Table 5: Clinical Activity Score classification

\begin{tabular}{|c|c|}
\hline Item & Parameters assessed \\
\hline \multirow{2}{*}{$\begin{array}{l}\text { Pain (over the last } 4 \\
\text { weeks) }\end{array}$} & Pain on or behind the globe \\
\hline & Pain on attempted eye movements \\
\hline \multirow[t]{2}{*}{ Redness } & Redness of the eyelid(s) \\
\hline & $\begin{array}{l}\text { Diffuse redness of the conjunctiva, covering at least } \\
\text { one quadrant }\end{array}$ \\
\hline \multirow[t]{4}{*}{ Swelling } & Swelling of the eyelid(s) \\
\hline & Chemosis \\
\hline & Swollen caruncle \\
\hline & $\begin{array}{l}\text { Increase of }>2 \mathrm{~mm} \text { proptosis during a period of } \\
1-3 \text { months }\end{array}$ \\
\hline \multirow{2}{*}{$\begin{array}{l}\text { Impaired function (over } \\
\text { the past } 1-3 \text { months) }\end{array}$} & Decrease in eye movements in any direction \\
\hline & $\begin{array}{l}\text { Decrease in visual acuity (1 or more lines on Snellen } \\
\text { chart, using a pinhole) }\end{array}$ \\
\hline
\end{tabular}

\section{Clinical features}

The clinical features of TED include proptosis, eyelid retraction, soft tissue signs, restrictive myopathy and optic neuropathy. Eyelid retraction (unilateral or bilateral in around $90 \%$ of individuals at some point) and proptosis (unilateral or bilateral in up to $60 \%$ of individuals) are the most common features of TED. ${ }^{52}$ Eyelid retraction occurs during the active stage due to sympathetic stimulation of Muller's muscle by thyroid hormone overload. While in the fibrotic stage, eyelid retraction is attributed to a contracture of the levator palpebrae superioris and inferior rectus along with fibrotic adhesion to surrounding structures. The levator palpebrae superioris may also have compensatory overaction due to hypotropia, and conversely, the inferior rectus may attempt compensation for hypertropia. On examination, lid retraction is presumed based on 'scleral show', a situation in which the upper lid margin is at or above the superior limbus. The lower lid margin normally rests at the inferior limbus. In the case of lid retraction, the margin lies below the limbus, allowing sclera to become visible. ${ }^{.54}$

Proptosis in TED is axial and frequently permanent. It can be symmetrical or asymmetrical in bilateral cases. ${ }^{6}$ Restrictive myopathy is seen in $30-$ $50 \%$ of patients. ${ }^{6}$ During the active stage, it occurs due to inflammation and muscle enlargement, and in the quiescent stage, due to fibrosis. Diplopia occurs in about $17 \%$ of patients. ${ }^{12}$ The order of involvement of muscles is usually inferior rectus, followed by medial rectus, superior rectus, and finally lateral rectus. ${ }^{6}$

Although blurred vision has been reported in around 7\% of patients with TED, optic neuropathy contributes to less than $2 \%$ of cases. ${ }^{12}$ The enlarged recti muscles and swollen orbital tissues cause compression of the optic nerve or its blood supply, and compromise its function. Such a compartment syndrome effect may occur even in the absence of significant proptosis and is called 'auto-decompression'. ${ }^{12}$ In these patients it is imperative to regularly monitor optic nerve function tests, such as visual acuity, red colour desaturation, RAPD, light brightness appreciation, visual fields and ophthalmoscopic examination of the optic disc.

\section{Eponymous signs in TED}

There are a few clinical signs that are classically associated with TED. ${ }^{54,55}$ These signs are listed below:

- von Graefe's sign: characterized by lid lag. As the patient looks down the upper lid does not follow the downward movement of the eye, as is evident by the increasing exposure of the sclera superiorly
- Dalrymple's sign: upper lid retraction in primary position

- Griffith's sign: lower lid lag on looking up

- Joffrey's sign: absence of folds on the forehead on looking up

- Boston's sign: jerky movements of the upper lid on looking down

- Stellwag's sign: infrequent blinking

- Kocher's sign: staring and frightened appearance, especially on attentive fixation

- Moebius' sign: convergence deficiency

- Gofford's sign: difficulty in inversion of the upper lid

- Sainton's sign: on looking up, the frontalis contracts after the levator palpebrae superioris has ceased to act

- Rosenbach's sign: a trembling of the lid evident on gentle closure of the eyelids

- Enroth's sign: fullness of eyelids due to puffy, oedematous swelling of the tissues

- Jellinek's sign: pigmentation of the upper lid

- Suker's sign: inability to maintain fixation on extreme lateral fixation of eyes

- Ballet's sign: partial or complete immobility of one or all extrinsic ocular muscles without an internal ophthalmoplegia

\section{Treatment of thyroid eye disease}

The treatment of proptosis is directed at the cause. A few general principles can be applied depending upon the severity of the proptosis and the effects produced by it. There is often dry eye produced by the inflammation, lagophthalmos and poor blink mechanisms. This can be managed by lubricants, cyclosporine and a short course of topical steroids, if required. In proptosis associated with exposure keratopathy, a moist chamber or tarsorrhaphy can be employed to tide over the condition. If the condition does not resolve, then later, decompressive surgery can be performed. In the case of retrobulbar haemorrhage, a small lateral canthotomy and application of ice over the eyelids should be done urgently to stop the bleeding and reduce the intra-orbital pressure that can compromise optic nerve function. Since TED is the commonest cause of proptosis in adults, a brief discussion on its management is provided below. ${ }^{34,47,53}$

The management of TED should be a multidisciplinary approach involving not only the ophthalmologist, but also the endocrinologist, neuro-surgeon, optometrist and nursing staff to take care of the patient. This disease is debilitating, vison-threatening, and affects quality of life. Smoking is four times more likely associated with the development and progression of TED. ${ }^{56}$ Therefore, cessation of smoking should be encouraged in all smokers. Low selenium levels are also found in some individuals, and supplementation with this micro-nutrient can be done in such patients. Radioactive iodine treatment tends to worsen TED, and patients undergoing such therapy should be covered with systemic steroids. Lubricants are useful in reducing the dry eye and grittiness associated with the condition. In mild cases, patients may only require this treatment. Patients with poor Bell's phenomenon should be supplemented with night-time use of lubricant ointment or gels. As TED has an inflammatory pathophysiology, soft-tissue involvement can be treated with cyclosporine eye drops. In case there is increased intraocular pressure on ocular movements, the patients should be treated with appropriate anti-glaucoma medications. Stable diplopia can be managed by the use of prisms or botulinum toxin. $6 ., 52$

In moderate to severe cases of TED, active disease must be treated with oral or intravenous corticosteroids. In case there is no sight-threatening emergency, a low dose of oral steroid ( 10 mg/day) for 4-6 weeks followed by a slow taper can be attempted. Intra-orbital injection of triamcinolone 
or dexamethasone is also effective. The effect of such treatment starts in 2-3 days and lasts a few weeks, when it can be repeated. In cases of sight-threatening optic neuropathy, intravenous methylprednisolone (500 mg-1 gm) is given weekly for 6-12 weeks. Steroid-sparing agents have also been reported. These include rituximab, etanercept, intravenous immunoglobulin, colchicine and teprotumumab, a human insulin-like growth factor I receptor inhibitory monoclonal antibody. ${ }^{12}$

Radioactive iodine therapy has been utilized in selected cases, when vision is not threatened. It is also used as steroid-sparing or steroidsupplementing therapy. The effect of radiation therapy starts at around 4-6 weeks and reaches maximum improvement by 4 months. Complications of radiation therapy include retinopathy (reportedly up to $20 \%$ at 10 years), cataracts (10-30\%), optic neuropathy, and increased risk of local cancers. ${ }^{12}$ Phosphorus-32 has been used as targeted brachytherapy and found to improve exophthalmos and to reduce tissue swelling and other associated symptoms. The procedure is cheaper and easier than radio-iodine therapy, and no side-effects have been reported over 3-12 months of follow-up. . $^{6,53}$

Any vision-threatening situation should be aggressively managed. In instances where steroids are ineffective and there is documented evidence of RAPD, visual field loss or a CT scan showing a crowded orbit, surgical decompression of the orbit is required. It can be done by one-wall (lateral wall) decompression, which can reduce 4-5 mm of proptosis; two-wall (lateral and medial combined); or three-wall (including the floor), which can reduce proptosis by 6-10 mm. In severe cases, the apex of the orbit is also resected. The greater the number of walls that are removed, the higher the risk of diplopia and enophthalmos. Fat-only decompression is performed in milder cases where visual function is not affected. It can also be combined when bony-wall decompression is performed to obtain better results, especially improvement in diplopia. ${ }^{6.12}$

Diplopia in primary or reading gaze requires surgical correction. However, the angle of deviation should be stable for 6-12 months. The commonest surgeries performed are inferior or medial rectus recession. Lid retraction surgery should be reserved as a last resort, as the recession of the inferior rectus can exacerbate lid retraction. In such cases, the recession of the levator palpebrae superioris or lower lid retractors is usually employed. Mullerotomy is also practiced for mild cases. Other modalities to manage lid retraction include injection of hyaluronic acid, triamcinolone or botulin toxin. These injections are often effective up to 6 months. ${ }^{53}$

\section{Conclusion}

Proptosis is often encountered in clinical practice, in situations ranging from the emergency room to the consultant's chambers. Evaluating a case of proptosis has to follow a systemic algorithm in order to avoid missing the likely diagnosis. In certain situations, time is of vital importance to prevent optic nerve compression and permanent loss of vision. This review provides a simplified pathway to approach proptosis and to improve patient care and outcomes. ${ }^{16} \mathrm{~J}$
Wright JE. Proptosis. Ann Roy Coll Surg Eng. 1970;47:323-34.

2. Dsouza S, Kandula P, Kamath G, Kamath M. Clinical profile of unilateral proptosis in a tertiary care centre. J Ophthalmol. 2017:2017:8546458.

3. Baujat B, Krastinova D, Bach CA, et al. Orbital morphology in exophthalmos and exorbitism. Plast Reconstr Surg. 2006; 117:542-52

4. Kokou V, Messan AK, Nidain M, et al. Proptosis: clinical and etiological features. New Front Ophthalmol. 2017;3:1-2.

5. Skuta GL, Cantor LB, Weiss JS. Orbits, Eyelids and Lacrima System. San Francisco: American Academy of Ophthalmology; 2011.

6. Kanski J, Bowling B. Orbit. In: Kanski J, Bowling B. Kanski's Clinical Ophthalmology 8th Edition: A Systematic Approach. Amsterdam, Netherlands: Elsevier, 2016;82-7.

7. Tan ST, Ashworth G, Czypionka S, et al. Vertical orbital dystopia. Plast Reconstr Surg. 1996;97:1349-61.

8. Warwick R. Eugene Wolff's Anatomy of Eye and Orbit. 6th Edition. London: WB Saunders \& Co., 1968:1-18.

9. Williams PL. Gray's Anatomy. 38th edition. Edinburgh: Churchill Livingstone, 2000;547-60.

10. Hiatt JL, Gartner LP. Textbook of Head and Neck Anatomy. 4th edn. Philadelphia: Wolters Kluwer Health, 2010;162-3.

11. Khurana AK, Khurana I. The skull, orbit and paranasal sinuses In: Anatomy and Physiology of Eye. 2nd edn. New Delhi: CBS Publishers and Distributors, 2006;487-92.

12. Nerad J, Smith T. Thyroid eye disease. In: El Toukhy EA (ed). Oculoplastic Surgery: A Practical Guide to Common Disorders. Switzerland: Springer Nature AG, 2020;491-98.

13. Vasu $K P$, Venugopal $M$. Etiological profile of proptosis - A prospective study. J Med Sci Clin Res. 2017;5:19608-12.

14. Mesud MZ, Babar TF, Iqbal A. Proptosis: etiology and demographic patterns. I Coll Physicians surg Pak. 2006:16:38-41.

15. Kaup S, Venkategowda HT. Clinical analysis of proptosis in a tertiary care hospital of South India. Int J Health Allied SC. 2017;6:149-54.

16. Topilow NJ, Tran AQ, Koo EB, Alabiad CR. Etiologies of proptosis: a review. Intern Med Rev (Wash DC). 2020;6:10.18103/im v6i3.852

17. Teja N, Reddy M, Vanama A. An etiological analysis of proptosis Int J Res Med Sci. 2015;3:2584-8.

18. Turnbull A, Trikha S, Whaley $C$, et al. Acquired unilateral proptosis - an overview of aetiology and radiological considerations. Available at: www.radmagazine.com/scientificarticle/acquired-unilateral-proptosis-an-overview-of-aetiologyand-radiological-considerations/ (accessed 26 January 2021)

19. Oakhill A, Willshaw H, Mann JR. Unilateral proptosis. Arch Dis
Child. 1981;56:549-51.

20. Sindhu K, Downie J, Ghabrial R, Martin F. Aetiology of childhood proptosis. J Paediatr Child Health. 1998;34:374-6.

21. Nambiar KR, Ajith PS, Arjunan A. Unilateral proptosis as the initial manifestation of malignancy. J Egypt Natl Canc Inst. 2017;29:159-61.

22. Chaudhry IA, Elkhamry SM, Al-Rashed W, Bosley TM. Carotid cavernous fistula: ophthalmological implications. Middle East Afr J Ophthalmol. 2009;16:57-63.

23. Rootman J. Distribution and differential diagnosis of orbital diseases. In: Diseases of Orbit. 2nd edn. Philadelphia: JB Lippincott; 1988.

24. Wade RG, Maddock TB, Ananth S. Orbital varix thrombosis: a rare cause of unilateral proptosis. BMJ Case Rep. 2013 Jan25;2013:bcr2012007935

25. Dolman PJ, Glazer LC, Harris GJ, et al. Mechanisms of visual loss in severe proptosis. Plast Reconstr Surg. 1991;7:256-60.

26. Gallego DF, Rivas-Grajales AM, Gallego CJ. Ocular auscultation: A review. Int J Med Students. 2015:3:102-6

27. Ogbeide E, Theophilus AO. Computed tomographic evaluation of proptosis in a Southern Nigerian tertiary hospital. Sahel Med J. 2015;18:66-70.

28. Viela MAP. Carotid cavernous fistula. Rev Bras Oftalmol. 2013;72:70-5

29. Ali MH, Jones S, Moss HE. Unilateral proptosis, redness, diplopia and numbness in a young woman. JAMA Ophthalmo 2016;134:1325-6.

30. Kim DJ, Song YJ, Kim SJ et al. Pituitary hemorrhage: Classification and related factors. J Korean Neurosurg Soc. 2009;46:23-30

31. Pereira TS, Kuniyoshi $\mathrm{CH}$, Leite $\mathrm{CA}$, et al. A comparative study of clinical vs. digital exophthalmometry measurement methods. $\int$ Ophthalmol. 2020;2020:1397410

32. Siakallis LC, Uddin JM, Miszkiel KA. Imaging investigation of thyroid eye disease. Ophthalmic Plast Reconstr Surg. 2018:34(4S Suppl. 1):S41-51.

33. Weiler DL. Thyroid eye disease: a review. Clin Exp Optom 2017;100:20-5

34. Kamminga N, Jansonius NM, Pott JW, Links TP. Unilateral proptosis: the role of medical history. Br J Ophthalmol. 2003;87:370-1.

35. Kawa MP, Machalińska A, Wilk G, Machalińska B. Graves' Ophthalmopathy Imaging Evaluation. In: Díaz-Soto G (ed) Thyroid Disorders: Focus on Hyperthyroidism. London, UK: InTech, 2014;141-58.

36. Gokharman D, Aydin S. Magnetic resonance imaging in orbita pathologies: a pictorial review. J Belg Soc Radiol. 2018:102:1-8.

37. Goh PS, Gi MT, Charlton A, et al. Review of orbital imaging. Eur
Radiol. 2008;66:387-95

38. Sahli E, Gunduz K. Thyroid-associated ophthalmopathy. Turk J Ophthalmol. 2017:47:94-105.

39. Gonçalves AC, Gebrim EM, Monteiro ML. Imaging studies for diagnosing Graves' orbitopathy and dysthyroid optic neuropathy. Clinics (Sao Paulo). 2012;67:1327-34.

40. Dallow RL. Evaluation of unilateral exophthalmos with ultrasonography: Analysis of 258 consecutive cases. Laryngoscope. 1975;85:1905-19.

41. Vlainich $A R$, Romaldini $J H$, Pedro $A B$, et al. Ultrasonography compared to magnetic resonance imaging in thyroidassociated Graves' ophthalmopathy. Arq Bras Endocrinol Metabol. 2011;55:184-8.

42. Lloyd GA. Orbital angiography. Proc R Soc Med. 1970;63:925-6.

43. Weatherhead RG. Pseudo-proptosis: A clinical classification of causes. Orbit. 1989;8:113-5.

44. Rizvi SAR, Gupta Y, Gupta M. Surgical treatment and histopathological analysis of proptosis. Nep J Oph. 2010;2:31-4.

45. Płoski R, Szymański K, Bednarczuk T. The genetic basis of Graves' disease. Curr Genomics. 2011;12:542-63.

46. Khalizadeh O, Noshad S, Rashidi A, Amirzargar A. Graves' ophthalmopathy: a review of immunogenetics. Curr Genomics. 2011;12:564-75

47. Lehmann GM, Feldon SE, Smith TJ, Phipps RP. Immune mechanisms in thyroid eye disease. Thyroid. 2008;18:959-65

48. Villanueva R, Inzerillo AM, Tomer $Y$, et al. Limited genetic susceptibility to severe Graves' ophthalmopathy: no role for CTLA- 4 but evidence for an environmental etiology. Thyroid. 2000;10:791-8.

49. Sikder S, Weinberg RS. Thyroid eye disease: pathogenesis and treatment. Ophthalmologica. 2010;224:199-203.

50. Mourits MP, Prummel MF, Wiersinga WM, Koornneef L. Clinical activity score as a guide in the management of patients with Graves' ophthalmopathy. Clin Endocrinol. 1997;47:9-14.

51. Hodgson NM, Rajaii F. Current understanding of the progression and management of thyroid associated orbitopathy: A systematic review. Ophthalmol Ther. 2020;9:21-33.

52. Li Q, Ye H, Ding Y, et al. Clinical characteristics of moderateto-severe thyroid-associated ophthalmopathy in 354 Chinese cases. PLOS ONE. 2017;12:e0176064.

53. Li Z, Cestari DM, Fortin E. Thyroid eye disease: what is new to know? Curr Opin Ophthalmol. 2018;29:528-34.

54. Singh P. Eponymous signs of thyroid ophthalmopathy. DJO. 2019;30:87-8

55. Duke-Elder S. The ocular adnexa. In: Duke-Elder S (ed). System of Ophthalmology. London: Henry Kimpton, 1974;939-68.

Thornton $\rfloor$ Kelly $\mathrm{S}$, Harrison $\mathrm{R}$, et al. Cigarette smoking and thyroid eye disease: a systemic review. Eye. 2007;21:1135-45. 